\title{
Maximum-Likelihood Analysis Using TREE-PUZZLE
}

Maximum-likelihood (ML) analysis is a statistically well-founded and well-known method used in many scientific fields. Edwards and Cavalli-Sforza (1964) have proposed ML for phylogenetics and Felsenstein (1981) made it applicable for molecular sequences. Although the computation time needed for ML analysis is large, recently, the usage of ML methods has substantially increased and has become an important component in molecular sequence analysis and phylogenetics. The ML approach is appealing because it incorporates explicit models of sequence evolution, and it also allows statistical tests of evolutionary hypothesis (Page and Holmes, 1998).

The TREE-PUZZLE software (Schmidt et al., 2002) applies the ML principle combined with a fast tree search algorithm called Quartet Puzzling to reconstruct phylogenetic trees from biological sequences. The Quartet Puzzling Algorithm uses quartets, i.e., groups of four sequences, to reconstruct large trees guided by the ML values of the quartet tree topologies (Fig. 6.6.1).

Moreover, Likelihood Mapping (Strimmer and von Haeseler, 1997), a method for visualizing the phylogenetic content of multiple sequence alignment, is implemented in TREE-PUZZLE. Likelihood Mapping can also be used to evaluate the quartet support for relationships among groups of sequences.

Furthermore, TREE-PUZZLE implements several statistics to compare different tree topologies.

In this unit, the authors use an amino acid alignment to explain the main features of TREE-PUZZLE. The dataset comprises the elongation factors EF-Tu/ $1 \alpha$ and EF-G/2, two genes that have duplicated before the three domains of life, Eucaryota, Crenarchaeota, and Bacteria split (see Table 6.6.1; similar datasets were first studied by Iwabe et al., 1989). The example presented here should enable one to design one's own analysis.

Despite using a protein example, TREE-PUZZLE can analyze nucleotide and binary data (e.g., restriction data) as well.

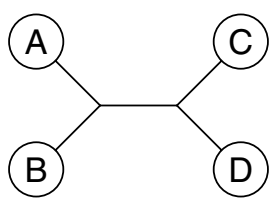

$A B \mid C D$

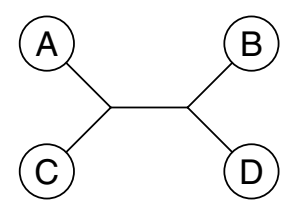

$\mathrm{ACIBD}$

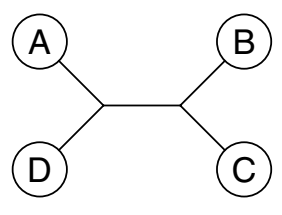

$A D I B C$

Figure 6.6.1 The three possible informative quartet tree topologies. 
Table 6.6.1 Sequences and Their Accession Numbers Used in the Test Dataset (EF . phy)

\begin{tabular}{llll}
\hline Sequence type & Identifier & Accession no. & Species name \\
\hline Bacterial EF-Tu & EFTU_ECOLI & P02990 & Escherichia coli \\
& EFTU_HELPY & P56003 & Helicobacter pylori \\
Crenarchaeotic EF-1 $\alpha$ (Archaea) & EFTU_MYCGE & P13927 & Mycoplasma genitalium \\
& EF1A_DESMO & P41203 & Desulfurococcus mobilis \\
& EF1A_SULAC & P17196 & Sulfolobus acidocaldarius \\
& EF1A_AERPE & Q9YAV0 & Aeropyrum pernix \\
Eucaryotic EF-1 $\alpha$ & EF11_HUMAN & P04720 & Homo sapiens \\
& EF12_MOUSE & P27706 & Mus musculus \\
& EF1A_CAEEL & P53013 & Caenorhabditis elegans \\
& EF1A_DICDI & P18624 & Dictyostelium discoideum \\
& EF11_DAUCA & P29521 & Daucus carota \\
Crenarchaeotic EF-2 (Archaea) & EF2_DESMO & P33159 & Desulfurococcus mobilis \\
& EF2_SULAC & P23112 & Sulfolobus acidocaldarius \\
& EF2_AERPE & Q9YC19 & Aeropyrum pernix \\
& EF2_HUMAN & P13639 & Homo sapiens \\
& EF2_MOUSE & P58252 & Mus musculus \\
& EF2_CAEEL & P29691 & Caenorhabditis elegans \\
& EF2_DICDI & P15112 & Dictyostelium discoideum \\
& EF2_BETVU & O23755 & Beta vulgaris \\
& EFG_ECOLI & P02996 & Escherichia coli \\
Bacterial EF-G & EFG_HELPY & P56002 & Helicobacter pylori \\
& EFG_MYCGE & P47335 & Mycoplasma genitalium \\
\hline
\end{tabular}

BASIC PROTOCOL 1

Maximum-

Likelihood Analysis Using TREE-PUZZLE

6.6.2

\section{RECONSTRUCT A PHYLOGENETIC TREE}

The main use of TREE-PUZZLE is to reconstruct phylogenetic trees from sequences. The example shows how to use TREE-PUZZLE to construct a tree from amino acid sequences assuming $\Gamma$-distributed rates across sites (UNIT 6.5).

\section{Necessary Resources}

Hardware

TREE-PUZZLE runs on Windows, Macintosh computers, and Unix/Linux systems including workstation clusters and parallel computers using parallel computing

\section{Software}

TREE-PUZZLE package (see Support Protocols 1 to 3 for information on how to obtain TREE-PUZZLE)

Files

Multiple Sequence Alignment file in standard PHYLIP format. The sample data set used (EF . phy) here is included with the TREE-PUZZLE software and on the Current Protocols Web site (http://www3.interscience.wiley.com/c_p/cpbi_ sampledatafiles.htm). 


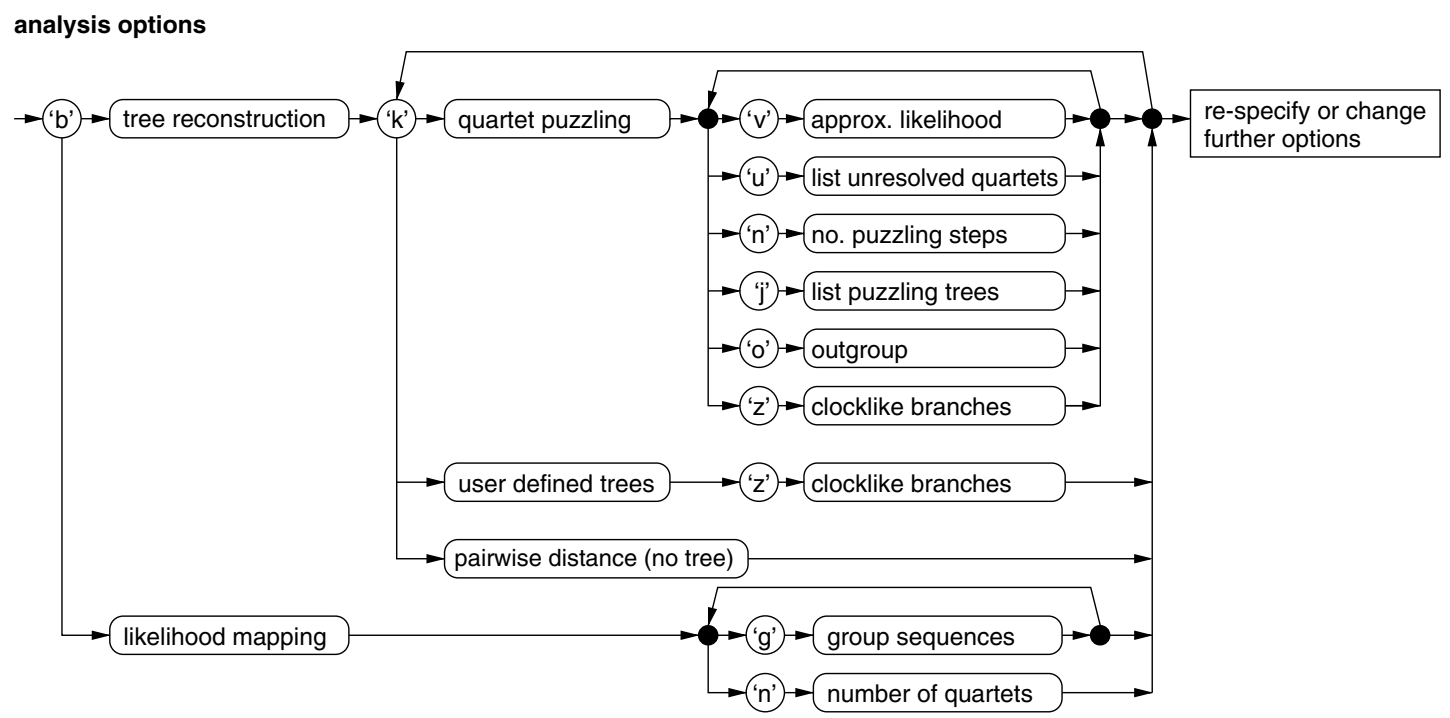

Figure 6.6.2 Flowchart of analysis type options in the TREE-PUZZLE menu. Options in TREE-PUZZLE are controlled by single letters. The flow chart shows the options that correspond to each letter. For example, entering the letter "b" toggles the analysis between tree reconstruction and likelihood mapping. Similarly, to choose among quartet puzzling, user defined trees, or pairwise distance matrices, enter the letter " $k$ " until the desired option is shown on the screen.

1. Obtain and install TREE-PUZZLE (see Support Protocols 1 to 3).

2. Change to the data directory in the TREE-PUZZLE directory and start the program with the command puzzle EF.phy.

Start puzzle in a terminal, e.g., MS DOS prompt (Windows) or xterm (APPENDIX 1C \& APPENDIX 1D; Unix/Linux), using the command puzzle alignmentfile, where alignmentfile is the name of the file containing the alignment to be analyzed, the example here is $\mathrm{EF}$. phy. If puzzle is invoked from a filemanager or without a filename, it will search for a file called infile in the current directory. If infile does not exist, TREE-PUZZLE will ask for a filename. The alignmentfile has to be in the current directory or the full path to its location must be given.

3. Change the type of analysis to tree reconstruction (using the "b" key) and the tree search procedure to quartet puzzling (using the " $k$ " key), if necessary (Fig. 6.6.2).

4. Adjust the outgroup to the sequence 22 EFG_MYCGE (using "o" and the number of the sequence).

By default, the first sequence is used to root the resulting tree for output. However, the root has no impact on the log-likelihood.

Note that the natural root lies between EF- $\alpha / T u$ and $E F-2 / G$ (Iwabe et al., 1989). Hence the output tree has to be rerooted using a phylogeny viewer.

For further discussion of selecting a tree root, see UNIT 6.1.

5. Choose parameter estimation to be performed approximately (with "e") using neighbor-joining trees (with " $\mathrm{x}$ ”).

Parameters are estimated using tree topologies. These are either inferred by neighbor-joining or given as usertree (usertree evaluation; see Basic Protocol 3). With the quartet samples + NJ option the evolutionary parameters are estimated on random quartet samples, neighbor-joining trees are only used for rate parameters. Approximate

Inferring

Evolutionary

Relationships

\subsection{3}

Supplement 1 
substitution model

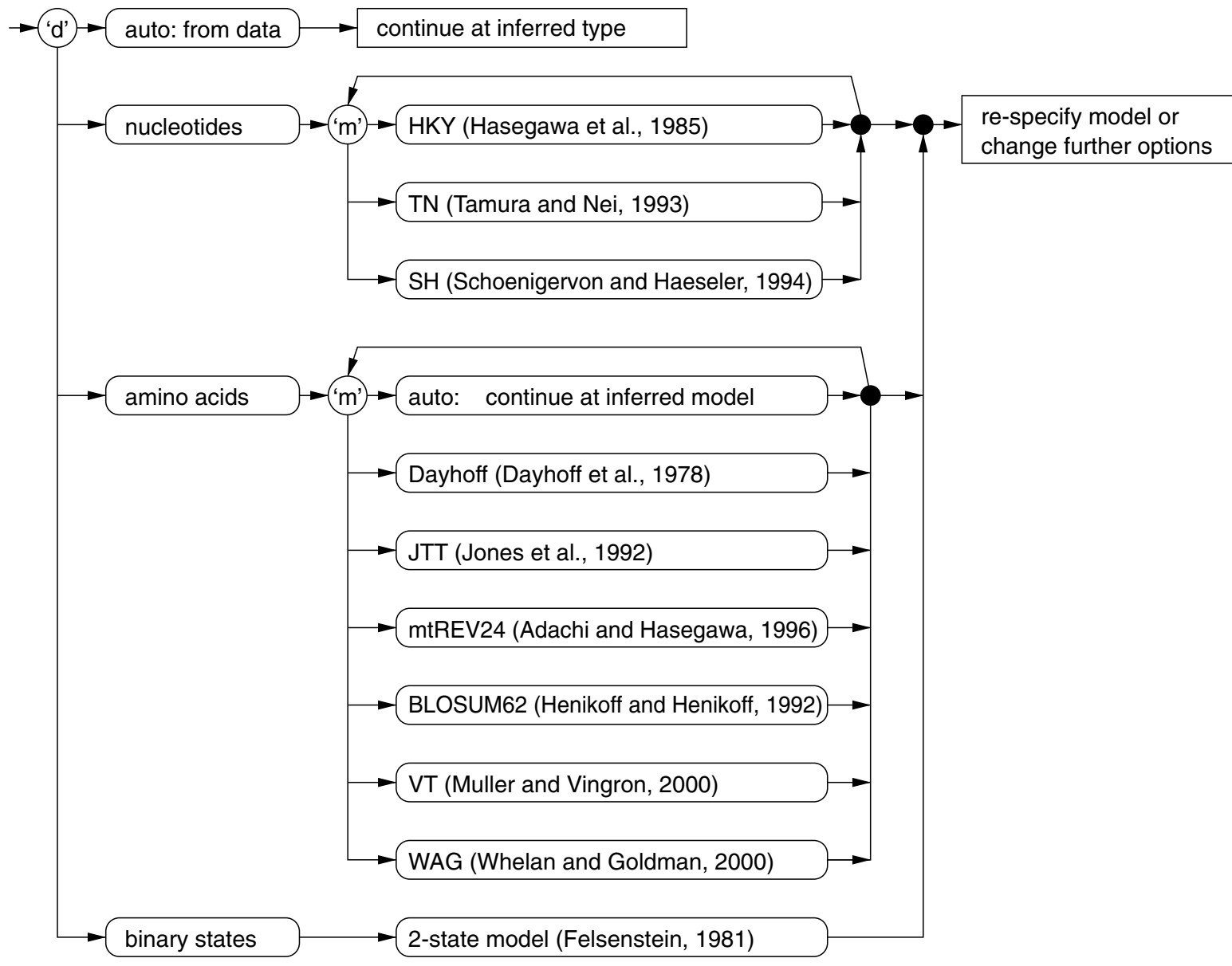

Figure 6.6.3 Flowchart of substitution model options in the TREE-PUZZLE menu.

estimation uses pairwise distances to fit the branch lengths of the tree topologies, while $M L$ branch lengths are inferred in the exact estimation.

\section{Choose a model of evolution}

6. Change the type of sequence data to amino acids (using "d") if the automatically assigned type is not correct (Fig. 6.6.3).

Using the character composition of the alignment, TREE-PUZZLE tries to figure out whether the type of data is nucleotide, protein, or binary data.

7. Choose an appropriate model of sequence evolution to analyze the dataset. For the example alignment, choose the VT model by entering "m" five times (Fig. 6.6.3).

Several models for protein evolution are implemented in TREE-PUZZLE. While the models by Dayhoff et al. (1978) and Jones et al. (1992) are universal models created from different protein families, more specific models are available, e.g., the mtREV24 model by Adachi and Hasegawa (1996) for mitochondrial protein sequences, whereas the VT (Müller and Vingron, 2000) and the WAG models (Whelan and Goldman, 2001) are suited to analyze distantly related sequences. The BLOSUM62 matrix (Henikoff and Henikoff, 1992; UNIT 3.5) was designed for database searches and thus should be used with caution for the analysis of evolutionary relationships. 
substitition process parameters

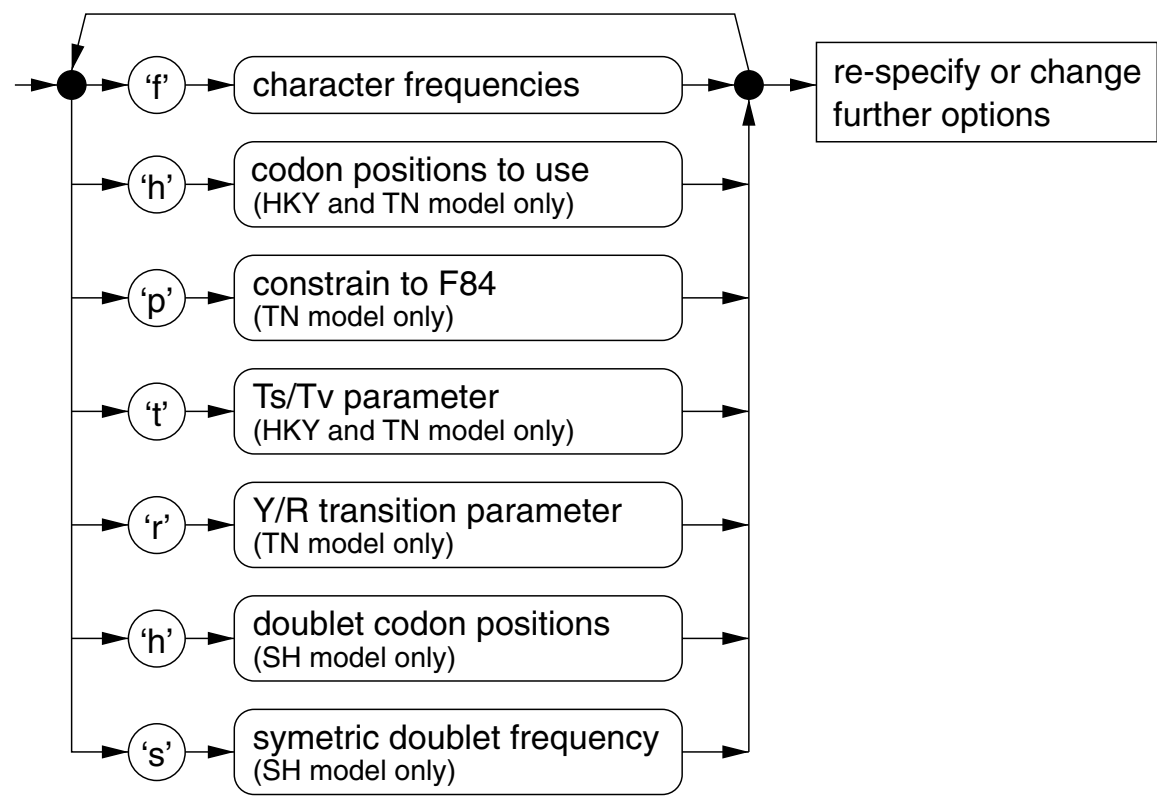

Figure 6.6.4 Flowchart of further substitution model parameters in the TREE-PUZZLE menu.

rate heterogeneity

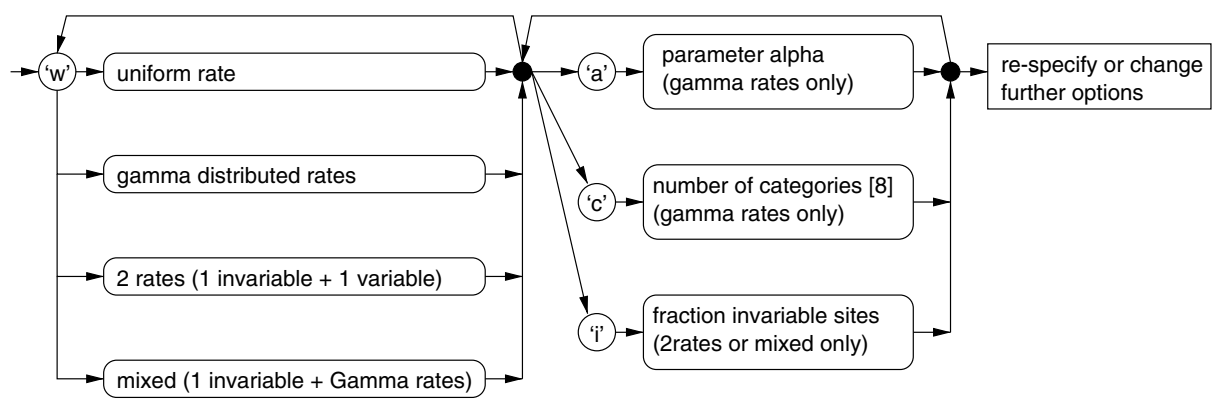

Figure 6.6.5 Flowchart of rate heterogeneity options in the TREE-PUZZLE menu.

For DNA (Fig. 6.6.4), the HKY (Hasegawa et al., 1985) and TN (Tamura and Nei, 1993) models are available. Those models can be restricted to simpler models like JC (Jukes and Cantor, 1969), K2P (Kimura, 1980), or F84 (Felsenstein, 1984) by setting substitution parameters accordingly (refer to the manual and UNITS 6.4 \& 6.5 for further details). Additionally, the SH nucleotide doublet model (Schöniger and von Haeseler, 1994) and a binary model based on the model of Felsenstein (1981) are implemented in TREE-PUZZLE.

8. Choose gamma-distributed rate heterogeneity model by typing "w" (Fig. 6.6.5).

It is known that positions in an alignment do not evolve with the same evolutionary rates, typically attributed to selective pressure or other functional constraints acting on positions

Inferring Evolutionary Relationships

\subsection{5}

Supplement 1 
parameter estimation

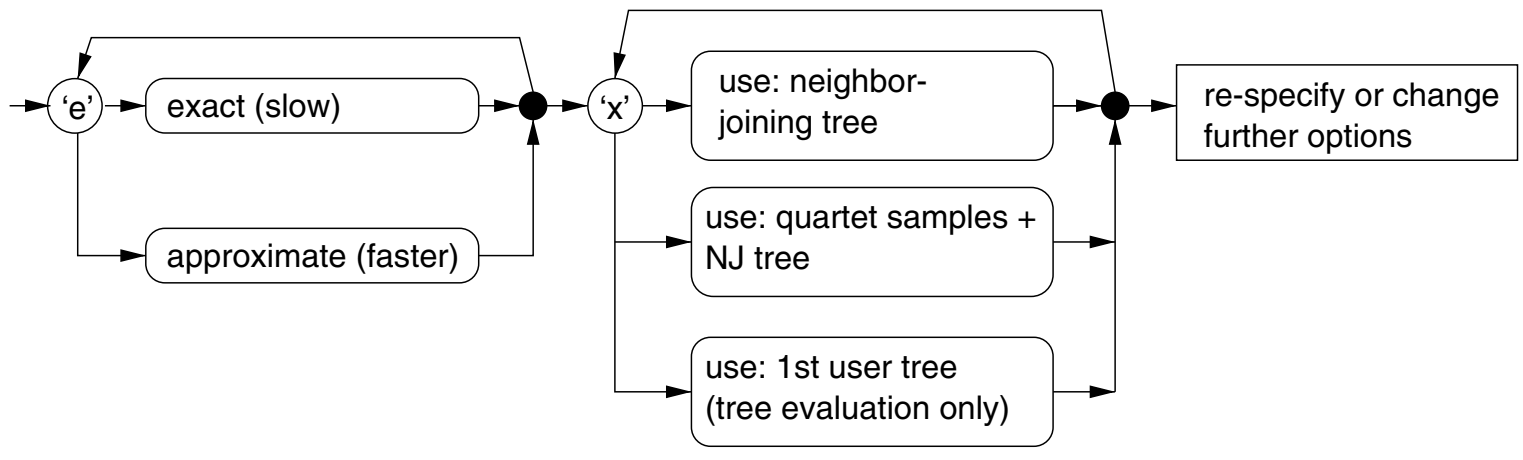

Figure 6.6.6 Flowchart of parameter estimation options in the TREE-PUZZLE menu.

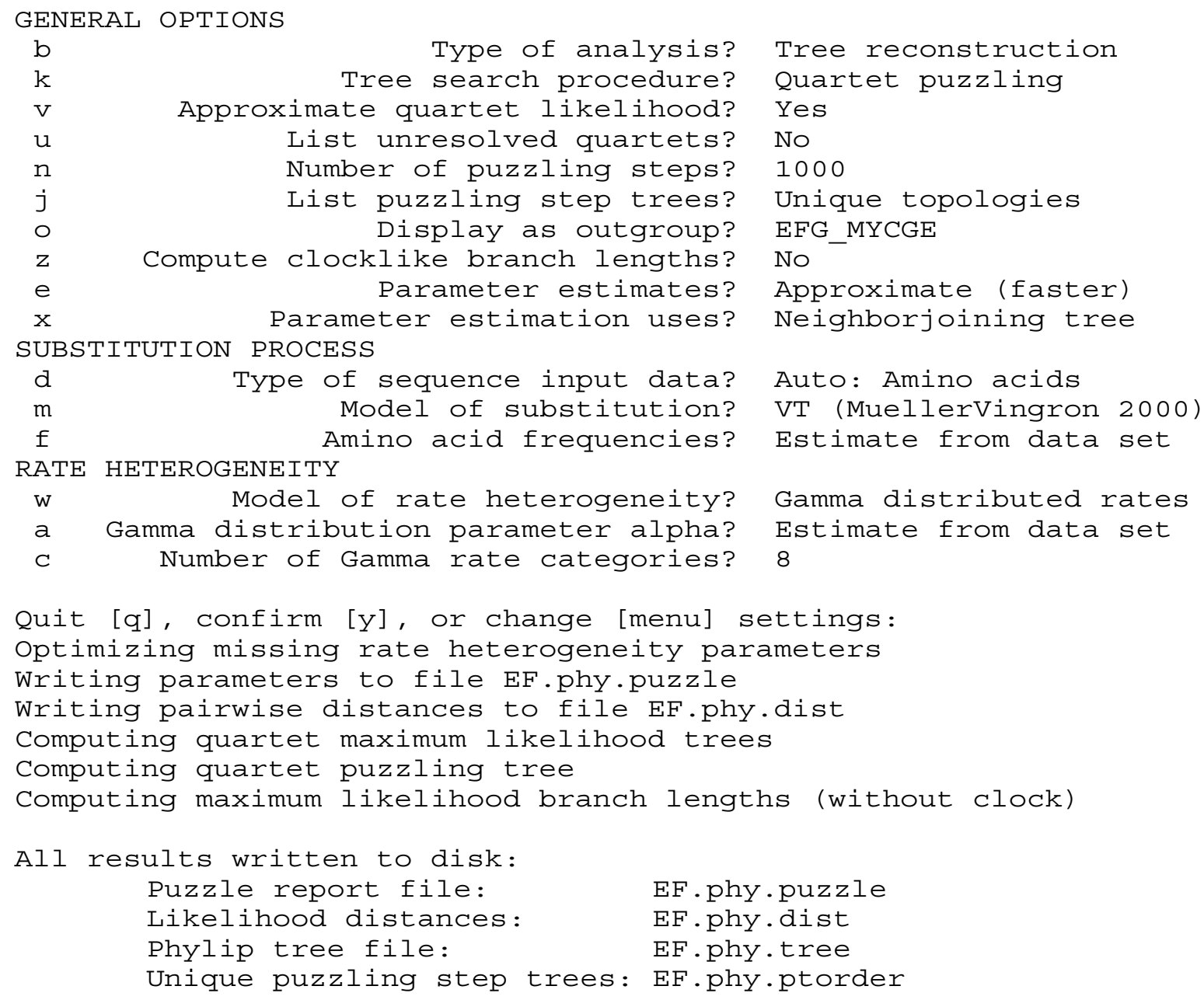

Quit [q], confirm [y], or change [menu] settings: Optimizing missing rate heterogeneity parameters

Writing parameters to file EF.phy.puzzle

Writing pairwise distances to file EF.phy.dist

Computing quartet maximum likelihood trees

Computing quartet puzzling tree

Computing maximum likelihood branch lengths (without clock)

All results written to disk:

Puzzle report file: EF.phy.puzzle

Likelihood distances: EF.phy.dist

Phylip tree file: EF.phy.tree

Unique puzzling step trees: EF.phy.ptorder

Figure 6.6.7 TREE-PUZZLE menu setting and screen output from tree reconstruction.

\subsection{6}




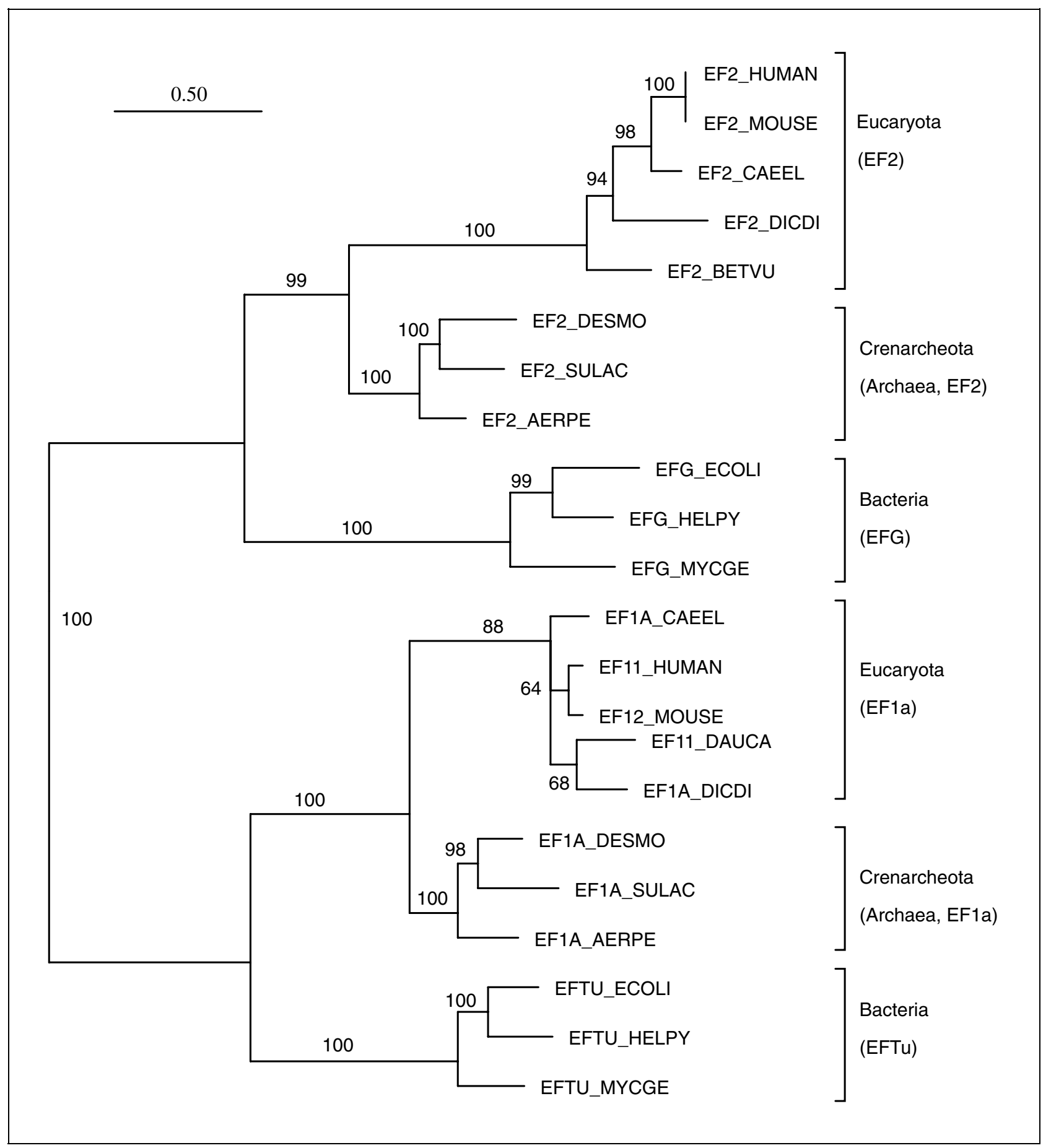

Figure 6.6.8 Phylogenetic tree reconstructed from the EF . phy dataset as described in Basic Protocol 1. The tree is rooted by the duplication event between EF-2/G and EF-1 $\alpha / T u$. 
of the sequence. In such cases, the assumption of rate heterogeneity can improve the estimation of the branch lengths.

Three different models of rate heterogeneity are implemented in TREE-PUZZLE. Besides gamma-distributed rates, there is the two-rates model that assumes a fraction of the positions to be invariable and a mixed model that considers the variable sites to evolve according to a gamma distribution. The amount of rate heterogeneity of the gamma-distributed rates is described by the shape parameter $\alpha$, where $\alpha<1$ describes strong heterogeneity, while large values describe homogeneity (for more details, refer to Gu et al., 1995; Page and Holmes, 1998; UNITS 6.4 \& 6.5).

If tree reconstructions with and without the assumption of rate heterogeneity construct different trees, those trees can be compared as described in Basic Protocol 3 to find out whether the resulting tree topologies are significantly different.

9. Set the list puzzling step trees option to unique topologies with the " $\mathrm{j}$ " key, to make TREE-PUZZLE write all (unique) intermediate tree topologies to file (EF . phy . ptorder or outptorder). When doing one's own analysis, it might be necessary to change other parameters.

Many other parameters and options can be set manually. For instance, it is possible to specify the amino acid or nucleotide composition. Figures 6.6.2 to 6.6.6 summarize all options currently available in TREE-PUZZLE. More details are given in the manual.

10. Start analysis by typing "y".

TREE-PUZZLE will now perform a tree reconstruction. During its run, it will indicate which steps are performed: first the missing parameters are estimated, then all possible quartet maximum-likelihood trees are computed, which are subsequently used to compute intermediate quartet puzzling trees. Finally, the likelihood and the branch lengths of the consensus tree are computed (Fig. 6.6.7).

\section{Examine the results}

11. Examine the puzzle report file. The report file is called EF.phy puzzle if the name of the alignment file was entered on the command line when the program was executed (e.g., puzzle EF.phy). Otherwise, the report is called outfile.

The puzzle report file presents the quality of the data as well as the reconstructed tree. Hence, it should be thoroughly examined (see Guidelines for Understanding Results below).

12. Examine the reconstructed tree by viewing the tree file EF. phy.tree (or outtree, Fig. 6.6.8) using a tree drawing program like TreeView or TreeTool (see UNIT 6.2 and Internet Resources below).

If a program cannot read such trees, it may be necessary to remove the leading comment (bordered by square brackets).

BASIC

PROTOCOL 2

Maximum-

Likelihood

Analysis Using

TREE-PUZZLE

6.6.8

ANALYZE THE CONTENT OF PHYLOGENETIC INFORMATION AND THE QUARTET SUPPORT FOR THE RELATIONSHIP OF GROUPS OF SEQUENCES

Likelihood mapping provides the opportunity to either check the content of phylogenetic information in an alignment or estimate the quartet support of relationships among groups of sequences. The former visualizes whether the data is suitable for phylogenetic analysis by measuring the resolution of the quartet topologies, trees of four sequences. This check should be run especially for large datasets to avoid spending days or maybe even weeks for phylogenetic analysis with data that have little phylogenetic information. For the latter method, one partitions a dataset into sets of two to four clusters. Likelihood mapping visualizes which of the possible relationships between these clusters is most supported by the reconstructed quartet tree topologies (Fig. 6.6.1). This method is also useful for 
reducing the runtime if the goal is to examine one special bipartition of a tree in a large dataset. The EF data (Table 6.6.1) will serve as an example. First, the suitability of the alignment for phylogenetic analysis is measured (step 4a). Second, the relationship of four subsets of the dataset (step $4 b$ ) is studied in more detail.

\section{Necessary Resources}

\section{Hardware}

TREE-PUZZLE runs on Windows and Macintosh computers as well as Unix/Linux systems including workstation clusters and parallel computers using parallel computing

\section{Software}

TREE-PUZZLE package (see Support Protocols 1 to 3 for information on how to obtain TREE-PUZZLE)

Files

Multiple Sequence Alignment file in standard PHYLIP format. The sample data set used here (EF . phy) is included with the TREE-PUZZLE software and on the Current Protocols Web site (http://www3.interscience.wiley.com/c_p/cpbi_ sampledatafiles.htm).

1. Obtain and install TREE-PUZZLE (see Support Protocols 1 to 3).

2. Change to the data directory in the TREE-PUZZLE directory and start puzzle with the command puzzle EF.phy.

Start puzzle in a terminal, e.g., MS DOS prompt (Windows) or xterm (Unix/Linux; APPENDIX IC \& APPENDIX 1D) using the command puzzle alignmentfile, where alignmentfile is the name of the file containing the alignment to be analyzed. If puzzle is invoked from a filemanager or without a filename, it will search for a file called infile in the current directory. If infile does not exist, TREE-PUZZLE will ask for a filename. The alignment file has to be in the current directory or the full path to its location must be given.

3. Change the type of analysis to Likelihood mapping (using the " $b$ " key).

4a. Leave the sequences ungrouped for a general likelihood mapping analysis to test the dataset.

4b. Group the sequences into four clusters (using "g"). Assign crenarchaeotic EF-2 to cluster a, bacterial EF-G to b, eucaryotic EF-2 to c, and all EF-1 $\alpha / \mathrm{Tu}$ sequences to cluster d (Table 6.6.1).

To analyze the phylogenetic content among clusters define two to four disjoint sets of sequences from the alignment by assigning each sequence the name of the cluster $\mathrm{a}, \mathrm{b}, \mathrm{C}$, or $\mathrm{d}$ (in the case of less than four clusters, $\mathrm{c}$ and/or $\mathrm{d}$ are not valid). Assigning $\mathrm{x}$ will exclude a sequence from the analysis. Each sequence must be labeled a, b, (c, d), or $\mathrm{x}$.

A two-cluster analysis will check for the quartet support for bipartition into the two clusters, whereas a four-cluster analysis will infer the quartet support for any of the three possible relationships of the four clusters, namely $(a b \mid c d),(a c \mid b d)$, or ( $a d \mid b c)$. Where "I" denotes the inner branch that separates the groups (Fig. 6.6.1).

\section{Choose a model of evolution (for more information, see Basic Protocol 1, steps 6 to 9)}

5. Change the type of sequence data (using "d") if the automatically assigned type is wrong.

TREE-PUZZLE should have set the data type correctly to amino acids for the example.

Inferring

Evolutionary

Relationships

6.6.9

Supplement 1 


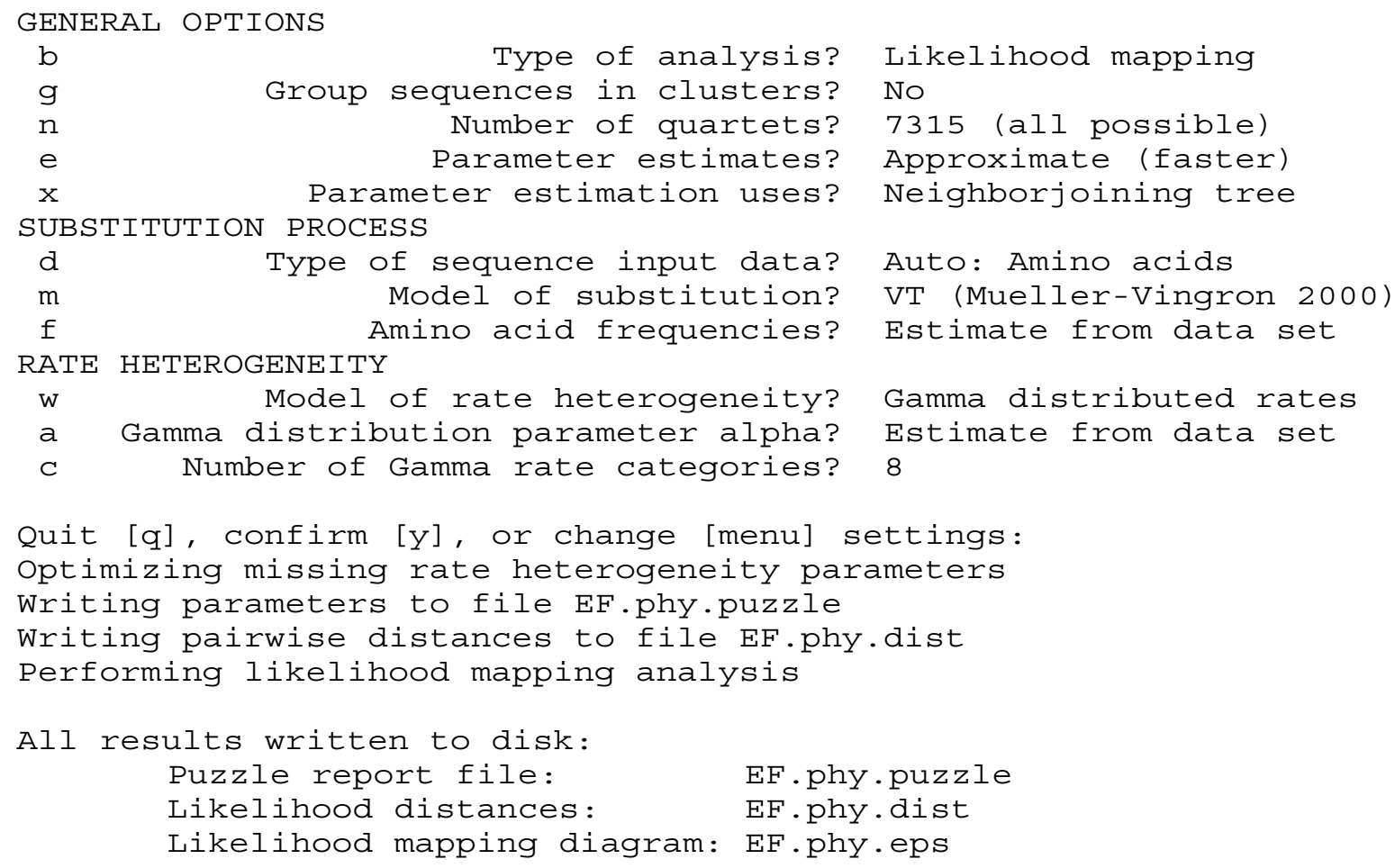

Figure 6.6.9 TREE-PUZZLE menu setting and screen output from likelihood-mapping analysis.

6. Choose an appropriate model of evolution to analyze a dataset. For the example alignment, choose the VT model by entering "m" five times.

7. Choose rate heterogeneity model by typing "w".

8. Change other parameters, if necessary. For the example, leave the parameters unchanged.

The number of quartets used in the analysis can be set by the $\mathrm{n}$ option. If the number of existing quartets is larger than the specified number, a random subset of all possible quartets is chosen by default, but the size of the sample is also adjustable.

9. Start analysis by typing "y".

TREE-PUZZLE will now perform a likelihood-mapping analysis. During the run, it will indicate which steps are performed: first the missing parameters are estimated, then the likelihood-mapping analysis is performed evaluating quartet maximum-likelihood trees. For large datasets, a random subset of quartets is analyzed (Fig. 6.6.9).

\section{Examine the results}

10. Examine the puzzle report file. The report file is called EF . phy . puzzle, if starting with the alignment file from the command line (e.g., puzzle.EF.phy), or out $\mathrm{file}$ if entering the alignment file manually.

The puzzle report file presents the quality of the data as well as the results of the likelihood mapping. Hence, it should be thoroughly examined.

Maximum-

Likelihood Analysis Using TREE-PUZZLE

6.6.10
11. Examine the likelihood-mapping diagram (Figs. 6.6.10, 6.6.11, and 6.6.12) EF.phy.eps (or outlm.eps) using a PostScript browser like ghostscript/ghostview (see Internet Resources). 

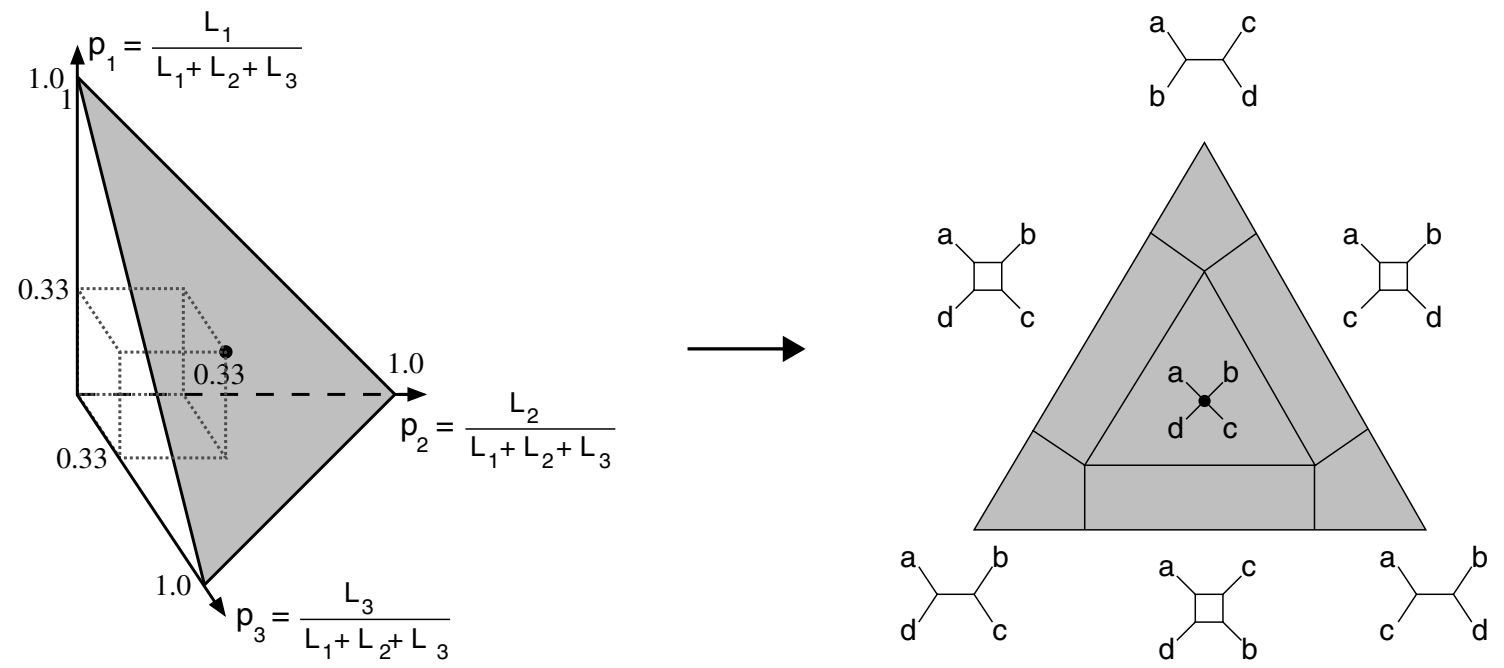

Figure 6.6.10 How likelihood weights are plotted in a likelihood-mapping diagram. Left side: likelihood weight plotted in a three-dimensional coordinate system. Right side: the simplex and its areas and the corresponding quartet topologies. The gray triangles are identical, only viewed from different angles.

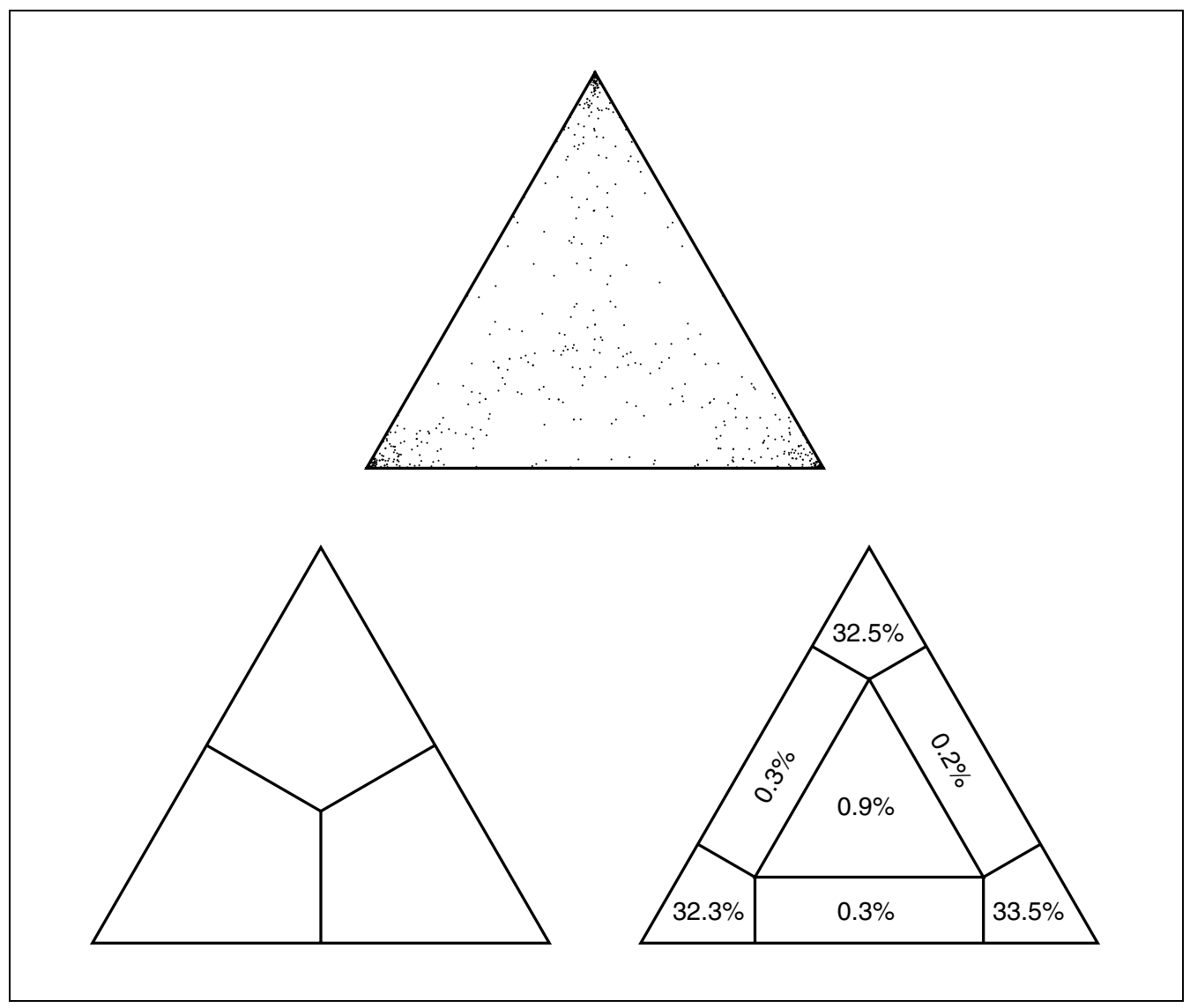

Figure 6.6.11 Likelihood-mapping diagram visualizing the phylogenetic content of the EF.phy dataset performed as described in Basic Protocol 2.

Inferring

Evolutionary

Relationships

\subsubsection{1}


(Crenarchaeota, Bacteria)-(Eucaryota, EF-1a/Tu)

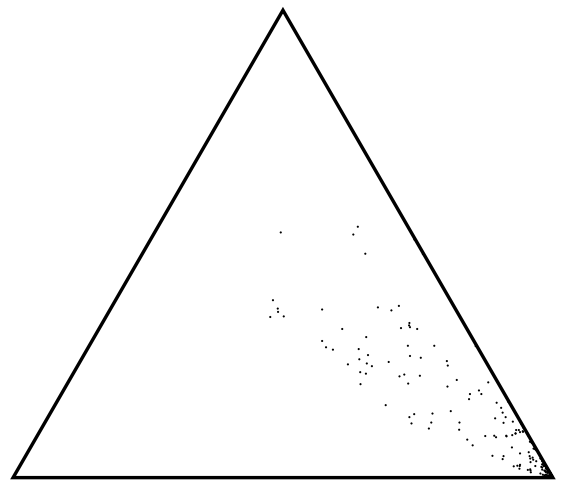

Crenarchaeota, EF-1a/Tu)-

(Bacteria, Eucaryota)

(Crenarchaeota, Eucaryota)-

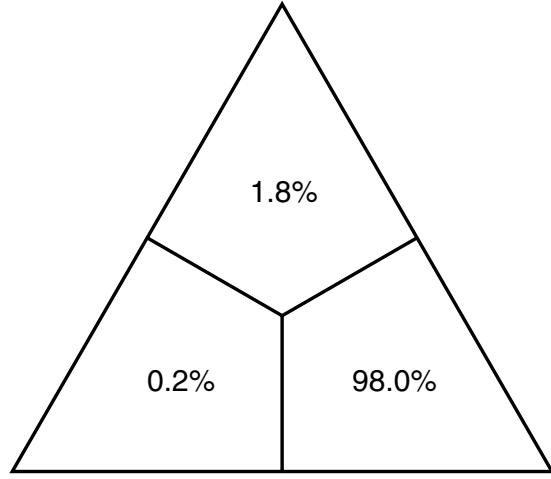

(Bacteria, EF-1a/Tu)

BASIC PROTOCOL 3

Maximum-

Likelihood Analysis Using TREE-PUZZLE

Figure 6.6.12 Likelihood-mapping diagram visualizing the support for a Crenarchaeota-Eucaryota sister group in the EF-2/G genes of the EF . phy dataset as described in Basic Protocol 2.

\section{COMPARE TREE TOPOLOGIES}

A third type of analysis implemented in TREE-PUZZLE is the likelihood-based comparison of two or more tree topologies using the tests suggested by Kishino and Hasegawa (1989), Shimodaira and Hasegawa (1999), and the so-called expected likelihood weights (Strimmer and Rambaut, 2002). These tests compare different trees to evaluate something like a confidence set of trees. The example used here is a dataset together with a set of trees with different branching patterns, comprising the tree reconstructed in Basic Protocol 1 and two trees with the different possible relationships of Crenarchaeota, Bacteria, and Eucaryota (Fig. 6.6.13)

\section{Necessary Resources}

Hardware

TREE-PUZZLE runs on Windows and Macintosh computers as well as Unix/Linux systems including workstation clusters and parallel computers using parallel computing

Software

TREE-PUZZLE package (see Support Protocols 1 to 3 for information on how to obtain TREE-PUZZLE) 


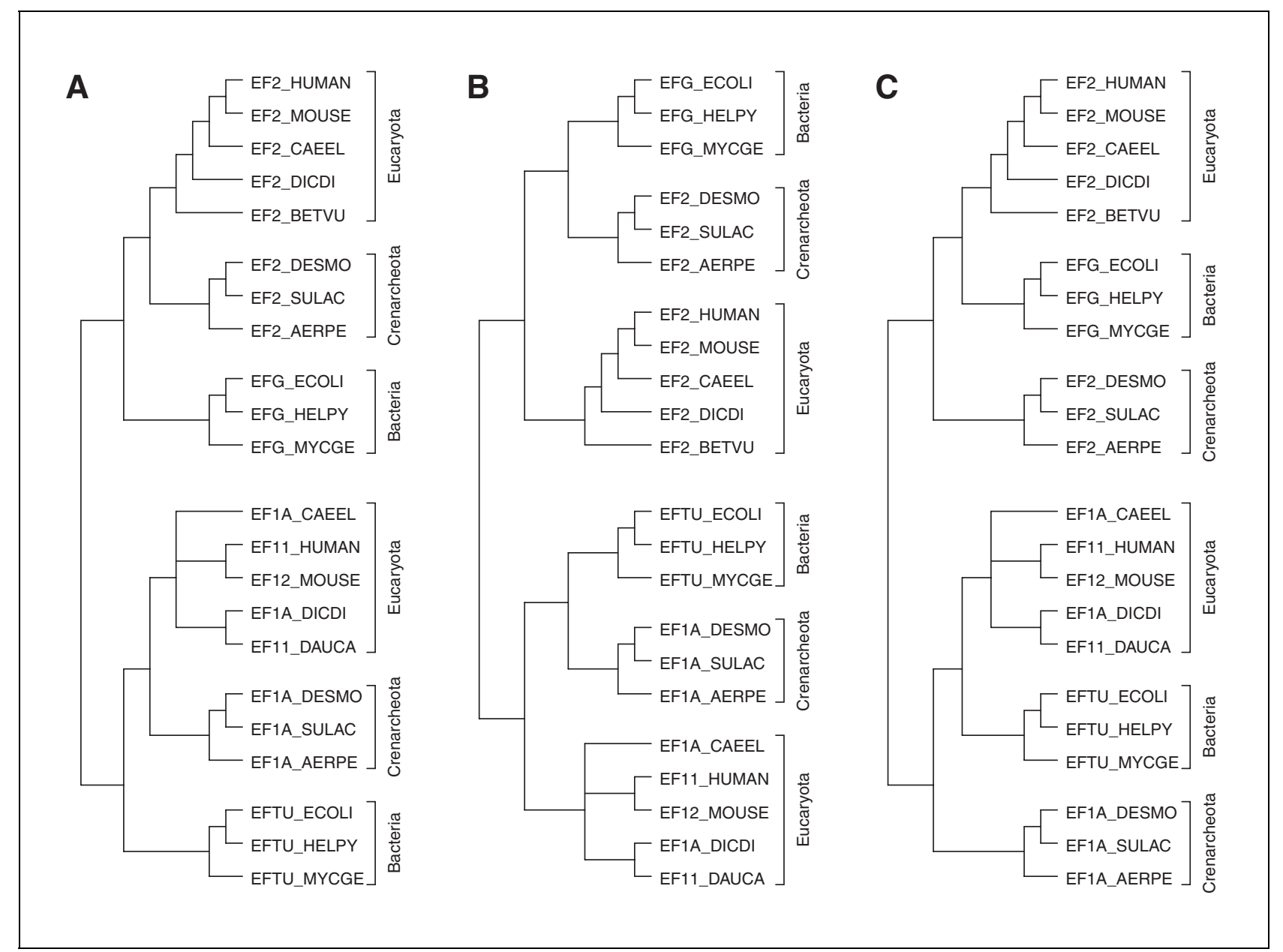

Figure 6.6.13 The three tree topologies used in the usertree comparison. (A) Tree 1: Eucaryota-Crenarchaeota sister groups, (B) Tree 2: Bacteria-Crenarchaeota sister groups, (C) Tree 3: Eucaryota-Bacteria sister groups. The tree topologies are used without branchlengths.

Files

Multiple Sequence Alignment file in standard PHYLIP format. A tree file containing the usertrees in PHYLIP tree format as produced by many programs like PHYLIP, TREE-PUZZLE, etc. (trees can span several lines and contain comments; for more information see UNIT 6.2); see file EF.3trees on the Current Protocols Web site at the URL below. The sample data set used below is included with the TREE-PUZZLE software and on the Current Protocols Web site (http://www3.interscience.wiley.com/c_p/cpbi_sampledatafiles.htm)

1. Obtain and install TREE-PUZZLE (see Support Protocols 1 to 3).

2. Change to the data directory in the TREE-PUZZLE directory and start puzzle with the command puzzle EF.phy EF.3trees.

Start puzzle in a terminal, e.g., MS DOS prompt (Windows) or xterm (Unix/Linux; APPENDIX 1C \& APPENDIX 1D) using the command puzzle alignmentfile usertreefile, where alignment $\mathrm{f} i \mathrm{le}$ is the name of the file containing the alignment to be analyzed and usertreefile is the name of the file that contains the tree topologies for comparison. If puzzle is invoked from a filemanager or without filenames, it will search for the files infile and intree in the current directory. If infile and/or intree does not exist, TREE-PUZZLE will ask for a filename. The alignmentfile and usertreefile have to be in the current directory or the full paths to their respective locations must be given.

Inferring

Evolutionary

Relationships

6.6.13

Supplement 1 


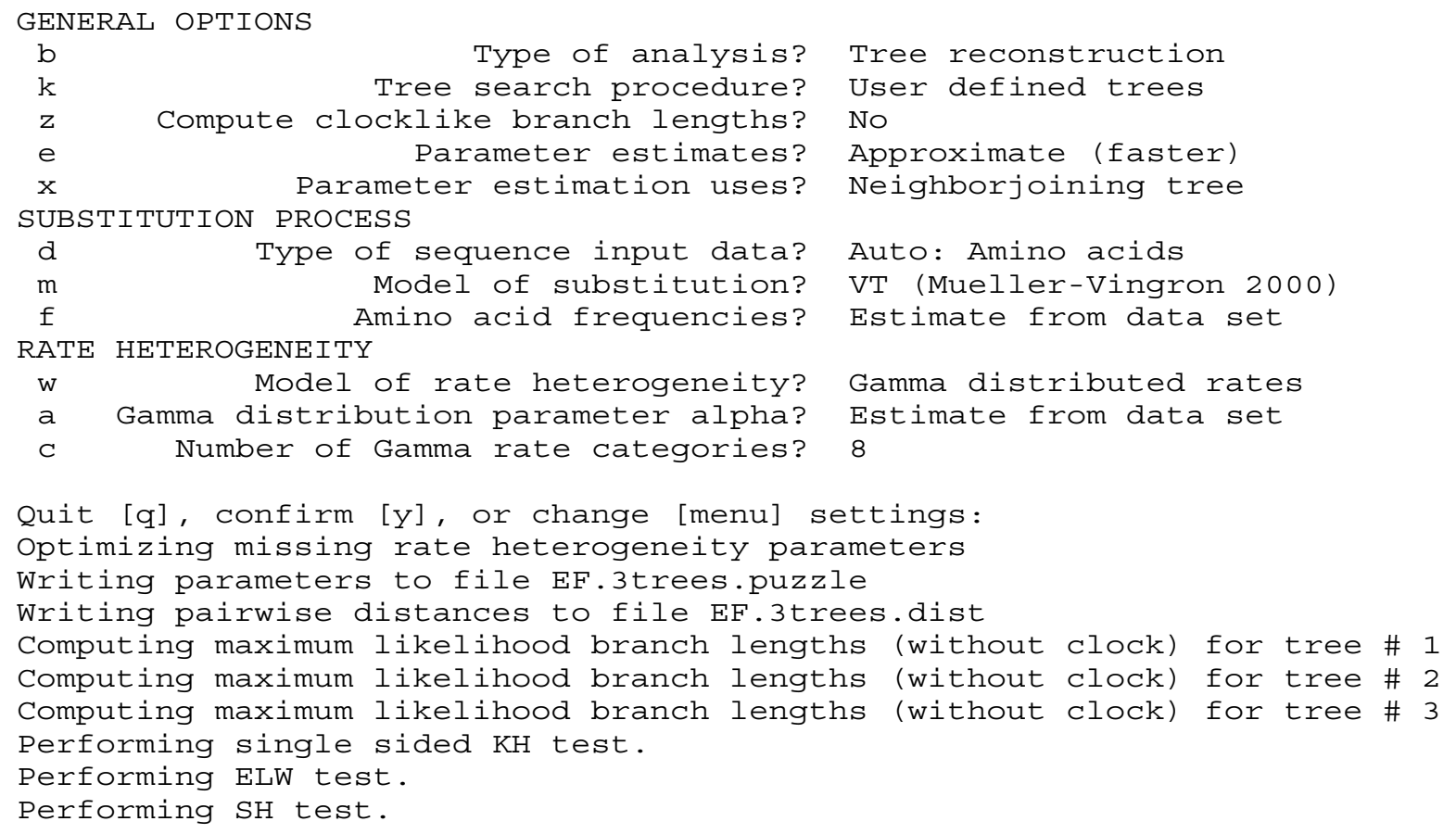

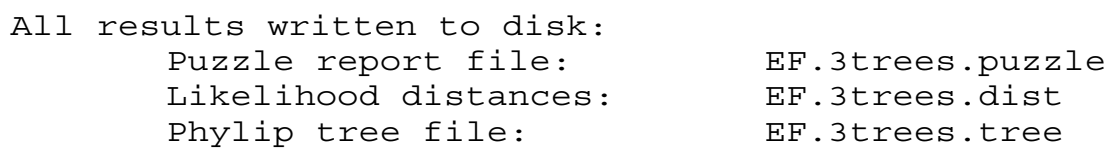

Figure 6.6.14 TREE-PUZZLE menu setting and screen output from usertree evaluation.

Maximum-

Likelihood Analysis Using TREE-PUZZLE

6.6.14
3. Change the type of analysis to tree reconstruction (using the "b" key) and the tree search procedure to user defined trees (using the " $k$ " key), if necessary.

4. Adjust the outgroup if necessary (using "o"). By default, the first sequence is used to root the resulting tree for output.

Choose a model of evolution (for more information, see Basic Protocol 1, steps 6 to 9)

5. Change the type of sequence data (using "d") if the automatically assigned type is wrong. TREE-PUZZLE should have set the data type correctly to amino acids for the example.

6. Choose an appropriate model of evolution to analyze the dataset. For this example alignment, choose the VT model by entering " $m$ " five times.

7. Choose rate heterogeneity model by typing "w".

8. Choose neighbor-joining (NJ) tree as the means for the parameter estimation with the " $x$ " key. Change other parameters, if necessary.

For tree evaluation, TREE-PUZZLE uses the first usertree for the parameter estimation by default. This makes sense for the evaluation of single trees, but to test a set of trees like in this example, a NJ tree should be used to estimate the parameters.

9. Start analysis by typing "y". 


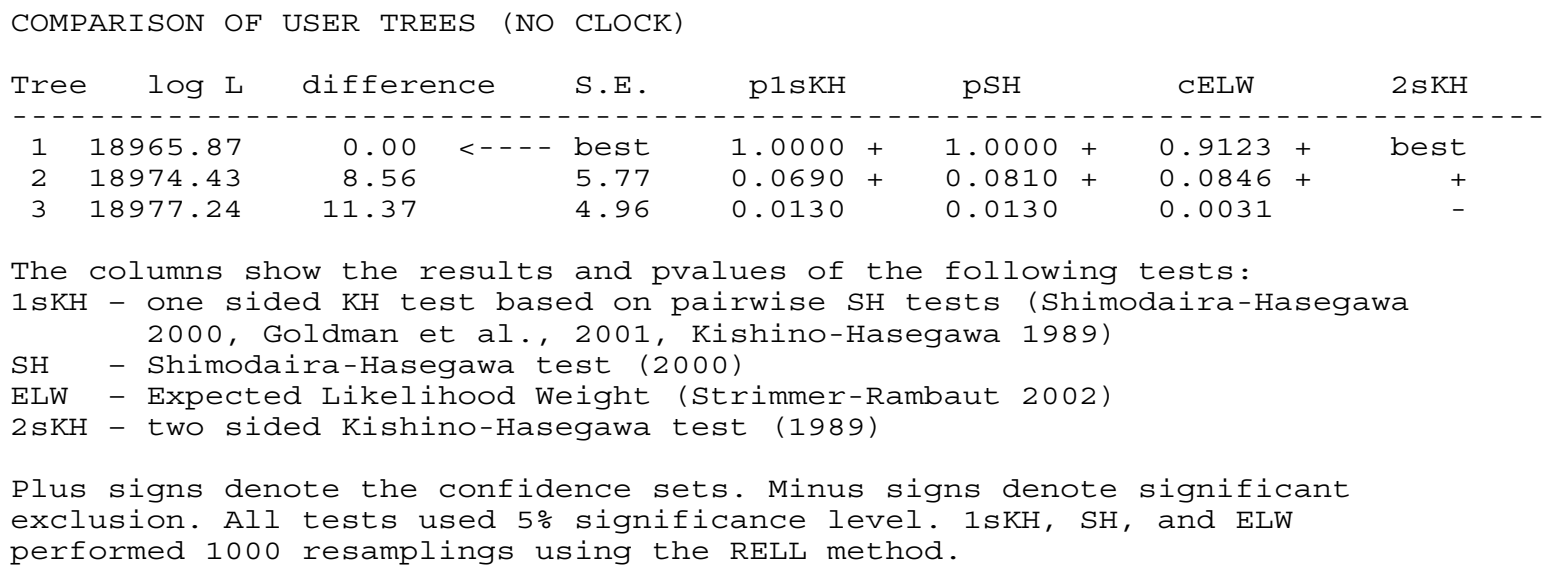

Figure 6.6.15 Results of the comparison of four trees from the EF . phy dataset as described in Basic Protocol 3.

TREE-PUZZLE will now evaluate and compare the tree topologies in the usertreefile (EF . 3trees). During its run, it will indicate which steps are performed: first, the missing parameters are estimated, then all trees in the usertreefile (EF . 3trees) are evaluated and the results are written to the puzzle report file (Fig. 6.6.14).

\section{Examine the results}

10. Examine the puzzle report file. The report file is called EF.3trees.puzzle, if starting with the alignment file from the command line, or out $f i l e$, if entering the alignment file manually.

The puzzle report file presents the quality of the data as well as the results of the usertree evaluation (Fig. 6.6.15). Hence, it should be thoroughly examined. The file $\mathrm{EF}$. 3 trees. tree (or outtree) contains each tree from the usertreefile in NEWICK tree format with estimated branch lengths. The trees can be viewed with tree drawing programs like TreeView or TreeTool (see UNIT 6.2 and Internet Resources). If a program cannot read such trees, it might be necessary to remove the leading comment (bordered by square brackets).

\section{OBTAIN AND INSTALL TREE-PUZZLE FOR UNIX/LINUX AND MacOS X}

This protocol describes how to obtain and install TREE-PUZZLE for Unix/Linux operating systems, including MacOS X.

\section{Necessary Resources}

\section{Hardware}

Unix/Linux system with TCP/IP Internet connection and a Web browser

\section{Software}

On Unix systems, an ANSI/ISO C compiler is needed, which is usually delivered with the operating system, otherwise, use the free GNU C compiler (http://www.gnu.org)

To use the parallel version of TREE-PUZZLE for supercomputers and workstation clusters, implementation of the MPI library (Message Passing Interface) is needed. There are several free implementations like LAM or MPICH (see http://www.lam-mpi.org/mpi/mpi/implementations for a list of implementations).

Inferring Evolutionary Relationships

\subsubsection{5}

Supplement 1 
1. Download the current TREE-PUZZLE package for Unix from http://www.treepuzzle.de. It has a name like tree-puzzle-X.X.tar.gz, where X.X should be the current version.

2. Unpack the package using:

gunzip tree-puzzle-X.X.tar.gz

tar-xvf tree-puzzle.X.X.tar

this should create a directory tree-puzzle-X.X. The subdirectories doc and data contain the manual and test data, respectively.

3. Change to the tree-puzzle-X.X directory.

4. Read the INSTALL file and the installation part of the manual carefully. Type the following commands to produce an executable:

. / configure

. /make

The command configure will determine the system type, and whether all needed software is installed. The make command will then compile the executable. If conf igure finds an MPI library installed, make will automatically produce the parallel version (ppuzzle) as well.

5. To install the executables, run the command:

make install

This will install the executables puzzle and ppuzzle (the parallel version).The programs will be installed to /usr/local/bin by default. If it is necessary to have the programs installed in another directory, change with conf igure (see the INSTALL file for more details) or copy the executables src/puzzle and/or src/ppuzzle to the desired location.

SUPPORT PROTOCOL 2

\section{OBTAIN AND INSTALL TREE-PUZZLE FOR MACINTOSH}

This protocol describes how to obtain and install TREE-PUZZLE for Macintosh operating systems, prior to MacOS X.

\section{Necessary Resources}

\section{Hardware}

Macintosh system with TCP/IP Internet connection and a Web browser

1. Download the current TREE-PUZZLE package for Macintosh from $h t t p: / / w w w$. tree-puzzle.de. It has a name like tree-puzzle-X.X.sit, where X.X should be the current version.

2. Unpack the package using a program like Stuffit (http://www.stuffit.com), which should belong to the MacOS release.

This should create a directory tree-puzzle-X.X, which contains the application treepuzzle-X. The subdirectories doc and data contain the manual and test data, respectively.

Maximum-

Likelihood Analysis Using TREE-PUZZLE 


\section{OBTAIN AND INSTALL TREE-PUZZLE FOR WINDOWS}

This protocol describes how to obtain and install TREE-PUZZLE for Windows operating systems.

\section{Necessary Resources}

\section{Hardware}

Windows system with TCP/IP Internet connection and a Web browser

1. Download the current TREE-PUZZLE package for Windows from $h t t p: / / w w w$. tree-puzzle.de. It has a name like tree-puzzle-X.X. zip, where X.X should be the current version.

2. Unpack the package using a program such as Winzip (http://www.winzip.com).

This should create a directory tree-puzzle-X.X. The subdirectories doc and data contain the manual and test data, respectively. In the bin directory, there is a Windows executable, puzzle.

3. Copy the Windows executable to the desired location.

This location should be in the Windows search path. For convenience, create a link on the Windows Desktop.

\section{GUIDELINES FOR UNDERSTANDING RESULTS}

\section{General Aspects}

As one can imagine, the outcome of an analysis is highly dependent on the data quality. In an optimal case, the data provides perfect phylogenetic information and no inconsistencies, and hence, the resulting tree will show the history of the sequences. Unfortunately, convergent evolution, multiple substitutions, and other processes introduce noise into the data. Thus, scrutinization of the data is necessary. TREE-PUZZLE tries to determine if the dataset is suited for phylogenetic analysis.

After running an analysis with puzzle, check the puzzle report file, called EF.3trees.puzzle (or outfile). TREE-PUZZLE measures several features of the dataset. In the SEQUENCE ALIGNMENT part, it shows the fraction of constant sites as well as how many different columns (site patterns) occur in the alignment. It also checks for identical sequences in the data. Identical sequences should be removed, because they increase computation time and provide no additional information about the phylogeny of the data.

TREE-PUZZLE also estimates the nucleotide composition or amino acid composition of the alignment. It tests if the composition of each sequence (e.g., amino acids or nucleotides) deviates significantly from the average composition. Also the amount of gaps and ambiguous characters, like " $\mathrm{N}$ " in nucleotide and " $\mathrm{X}$ " in protein sequences, is counted for each sequence. If a sequence contains many gaps and ambiguous characters, there might not be enough informative characters left to ensure a reliable placement of this sequence in the reconstructed tree.

These features of the data as well as the resolution of the quartets described below will help one to find out, which of the sequences might have caused inconsistencies in the analysis (see below).
Inferring Evolutionary

Relationships

\subsubsection{7}

Supplement 1 


\section{Tree Reconstruction (see Basic Protocol 1)}

To reconstruct phylogenies, TREE-PUZZLE applies a three-step algorithm called Quartet Puzzling (Strimmer and von Haeseler, 1996). In the first step, the maximum-likelihood step (ML step), all possible groups of four sequences, quartets, and their three different topologies (Fig. 6.6.1) are evaluated to create a set of quartets supported by the data. This step also takes into account if two or even all three tree topologies are almost equally good, i.e., partly resolved or unresolved topologies, respectively (Strimmer et al., 1997). Fully, partly, and unresolved quartets are explained in more detail below (see Likelihood Mapping for Data Quality and Quartet Support of Clusters). In the puzzling step, the supported quartet tree topologies are combined into an overall tree. Since this step is dependent on the input order, it is performed many times for randomized input orders, thus producing a large number of so-called intermediate or puzzle trees. These trees and their frequency can be output to file using the $j$ option as explained in Basic Protocol 1 (see manual for more details; Figure 6.6.2). In the final consensus step, a consensus tree is computed from the intermediate trees, which is then used to infer maximum-likelihood branch lengths and the maximum-likelihood value for the tree as described in Felsenstein (1981). The percentage of splits (i.e., bipartitions of the dataset induced by an internal edge in a tree) that occurred in the collection of intermediate trees is used as a reliability measure for the splits in the consensus tree. The higher these so-called support values, the more confidence one might put into the according bipartition. However, never confuse support values with bootstrap values.

If a split does not occur in $>50 \%$ of the intermediate trees it is not included in the consensus tree (McMorris and Neumann, 1983). Thus, multifurcations are possible. There is a multifurcation in the eucaryotic $\mathrm{EF} / 1 \alpha$ subtree in Figure 6.6.8.

In the puzzle report file, all intermediate trees occurring more often than 5\% are listed.

Moreover, in the puzzle report file (EF.phy.puzzle or outfile), the amount of fully, partly, and unresolved quartets for the entire dataset is shown. TREE-PUZZLE also outputs how frequently each sequence occurs in fully, partly, and unresolved quartets. This is another way of displaying phylogenetic information in the data (see Likelihood Mapping below) as well as in any of the sequences. If the reconstructed tree is highly unresolved, the unresolved quartets indicate whether the dataset was not suitable for tree reconstruction (overall fraction of unresolved quartets high) or if there are sequences that should be excluded because they introduce unresolved quartets. If the amount of unresolved quartet for a sequence is high, this sequence should be discarded from the dataset (see below for more details on unresolved quartets).

If the assumption of rate heterogeneity is applied, as in the example, then the report file also displays the site specific rates of each alignment site (RATE HETEROGENEITY section in the puzzle report file).

\section{Likelihood Mapping for Data Quality and Quartet Support of Clusters (see Basic Protocol 2)}

Likelihood mapping (Strimmer and von Haeseler, 1997) is based on likelihood values inferred for each of the three possible tree topologies for a quartet (Fig. 6.6.1). Every likelihood value is transferred into a weight (posterior probability), by dividing it by the sum of all three likelihoods (Strimmer et al., 1997). If one of the topologies has a higher likelihood than the others, its weight will be near 1.0 while the other weights are almost

Maximum-

Likelihood Analysis Using TREE-PUZZLE

6.6.18 
likelihood weights for a quartet add up to 1.0 and can be plotted in a three-dimensional coordinate system, one axis for each quartet topology. Each point falls into a triangular surface between $(1.0,0.0,0.0),(0.0,1.0,0.0)$, and $(0.0,0.0,1.0)$, as shown on the left side of Figure 6.6.10. Likelihood mapping plots the likelihood weights directly into such a triangle, also called simplex (Fig. 6.6.10, right side).

The likelihood mapping output (Figs. 6.6.12 and 6.6.14) comprises two different illustrations of the distribution of quartet weights in the simplex. One simplex is divided into three areas. Each area represents the region where a maximum-likelihood reconstruction would reconstruct the tree at the corner of the simplex. The second simplex is partitioned into seven regions. The central region represents the area of unresolved quartets. The three rectangles illustrate partly resolved quartets and the three trapezes reflect fully resolved quartets, defined by the trees in the corner (Fig. 6.6.10).

In an unrestricted likelihood mapping, all quartets are used for analysis, whereas in a grouped analysis, quartets are chosen according to the 2 to 4 assigned clusters:

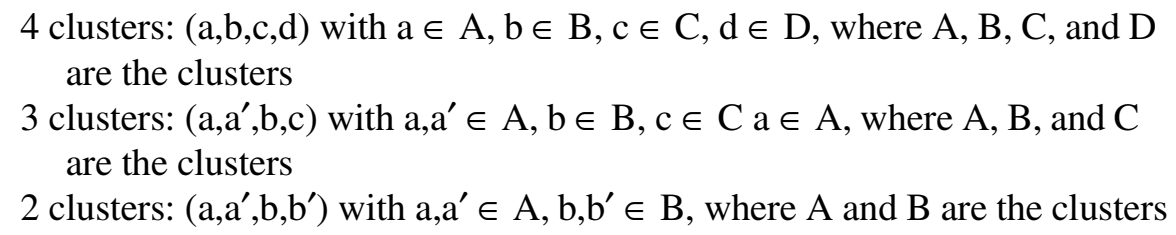

The results of the two likelihood mapping analyses are given in Figures 6.6.11 and 6.6.12. Figure 6.6.11 shows that the EF dataset is well suited for phylogenetic analysis with $98.3 \%$ fully resolved, $0.8 \%$ partly, and only $0.9 \%$ unresolved quartets. A large percentage of unresolved quartets would indicate that the data is not appropriate for phylogenetic analysis.

The analysis of the branching pattern within the EF-2/G sequences (Fig. 6.6.12) shows a preference for a monophyly of Crenarchaeota and Eucaryota. A percentage of $89.9 \%$ of all admissible quartets support this monophyly strongly (lower right simplex) and $98.0 \%$ of all quartets would suggest this tree, if the maximum-likelihood values of the quartet trees are considered.

\section{Comparison of Different Tree Topologies (see Basic Protocol 3)}

As mentioned above, the ML framework allows the test of competing hypotheses. Several tests have been proposed to compare phylogenetic trees (for a review, see Goldman et al., 2000). Three tests are implemented in TREE-PUZZLE.

The most commonly used is the pairwise KH test (Kishino and Hasegawa, 1989). This test is frequently used to compare the best tree, according to its ML value, to the other trees in the set.

Shimodaira and Hasegawa (1999) proposed a non-parametric test that is applicable if the maximum-likelihood tree, i.e., the tree with the highest likelihood, is an element of the collection of trees. Note that in a typical application, it is not ensured that an actual ML tree was found. Contrary to the $\mathrm{KH}$ test, which is essentially a pairwise test, the $\mathrm{SH}$ test compares all candidate trees simultaneously.

Recently, Strimmer and Rambaut (2002) published a method to infer confidence sets from possibly misspecified trees based on expected likelihood weights (ELW).

Before interpreting the results of the tests for one's own data, carefully studying the relevant literature and the limitations of each method are suggested. When performing 
tests on trees, make sure that these tests are applicable. Goldman et al. (2000) explain which tests are valid for a given dataset. According to Goldman et al. (2000), KH tests should not be applied if trees were constructed on the basis of the alignment that is then in turn used to compare the ML tree against the second and third best tree topology. The Shimodaira-Hasegawa test (1999) is a valid test if the best tree is in the test set and the test can be applied for a collection of trees. Whereas $\mathrm{KH}$ is a pairwise test, testing whether a tree is significantly worse than the best tree. The SH test is typically more conservative. It also has the tendency to depend on the number of trees in the test set, i.e., the larger the test set, the larger the confidence set. For more details about topology testing, especially for $\mathrm{KH}$ and SH tests and their applicability, refer to Goldman et al. (2000).

Basic Protocol 3 tests the following trees:

Tree 1: Eucaryota-Crenarchaeota sister groups for $\mathrm{EF}-2 / \mathrm{G}$ and $\mathrm{EF}-1 \alpha / \mathrm{Tu}$ (Fig. 6.6.13A)

Tree 2: Bacteria-Crenarchaeota sister groups for EF-2/G and EF-1 $\alpha / \mathrm{Tu}$ (Fig. 6.6.13B)

Tree 3: Eucaryota-Bacteria sister groups for EF-2/G and EF-1 $\alpha / \mathrm{Tu}$ (Fig. 6.6.13C).

The branching orders within the kingdoms are identical to Figure 6.6.8. The test results from the puzzle report file are given in Figure 6.6.15. All tests inferred "confidence sets" comprising trees 1 and 2. Note that tree 2, which groups together Bacteria and Crenarchaeota, got a lower likelihood, but is not significantly worse.

If all puzzling step trees occurring in Basic Protocol 1 are evaluated and tested together with the tree from Figure 6.6.8, the best tree found has a log-likelihood of -18958.52 compared to a log-likelihood of -18965.87 for the tree in Figure 6.6.8. The increase in likelihood is due to the fact that the best tree is fully resolved. This increase in the number of parameters (branches in the tree) leads to a higher likelihood. However, both statistical tests $(\mathrm{KH}$ and $\mathrm{SH})$ indicate that the Figure 6.6.8 tree is not worse than the best tree. Incidentally, the best tree is the most frequent tree among all intermediate trees.

\section{COMMENTARY}

\section{Background Information}

Most of the background information needed to understand the results as well as to interpret the data were discussed in the section Guidelines for Understanding Results above.

Programs that aim to reconstruct large phylogenetic trees have to contend with the enormous number of possible trees (Felsenstein, 1978). TREE-PUZZLE tries to cope with that problem by dividing the task into small fractions, the quartets (Strimmer and von Haeseler, 1996). For four sequences, only three informative topologies exist (Fig. 6.6.1) and the ML evaluation of each quartet is fast. Although there is still a large number of quartets to evaluate, this is often faster than computing likelihoods for a large number of large trees. From all quartet topologies, those chosen are the ones that are best supported by the data. TREE-PUZZLE takes into account that two or even all three topologies may be equally good (Strimmer et al., 1997). The set of quartet topologies are then "puzzled" together into so-called intermediate trees repeatedly with different orders of taxa. The set of intermediate trees offers two important advantages. The frequency of bipartitions found in the intermediate trees gives a reliability measure for the internal branches in the final tree without the necessity of running a large number of initial analyses. On the other hand, this set of somehow biologically reasonable trees, gives insight into the set of trees that is supported by the data.

The use of quartets also serves other purposes. The quartets are used to visualize the tree-likeness and subsequently the quality of the dataset for phylogenetic analysis. The number of unresolved quartets also helps to identify problematic sequences in the data sets. 
Another advantage is the broad variety of evolutionary models implemented. Besides DNA and binary sequence models, TREEPUZZLE offers several general and specialized models to reconstruct phylogenies from amino acid sequences, which are supported only by a very limited number of phylogenetic software.

\section{Critical Parameters}

\section{Number of sequences and length of the alignment}

As previously mentioned, the example dataset contains of 22 sequences and thus the reliability of the reconstructed topology depends heavily on the selection of species, which is very small for the evolutionary span it covers. Several researchers (e.g., Hillis, 1996) suggest that an increased number of sequences will increase the accuracy of the reconstructed tree. Another crucial point, that deserves attention is the length of the alignment. The authors' sample alignment is 915 amino acids long. If longer sequences were available, the accuracy of the reconstructed tree would increase and also the estimation of the parameters of evolution would be more precise.

\section{Model selection}

All phylogenetic methods rely on assumptions about the process of DNA or amino acid substitutions. The confidence one puts into a phylogenetic analysis depends on the goodness of fit, i.e., how appropriate is the model to describe the data. In a statistical framework, the goodness of fit is typically explored applying a likelihood ratio statistics. When the models are nested, i.e., the null model is a special case of the alternative model, the differences in the log-likelihood between both models is typically $\chi^{2}$ distributed. To select the best model, a variety of programs is available, e.g., ModelTest (Posada and Crandall, 1998; UNIT 6.5), which is applicable for DNA sequences. This program can be used to find the best model for a fixed tree topology. If, however, the tree topologies are different (the models are not nested), one needs to apply Monte Carlo simulations as suggested by Goldman (1993a,b).

\section{Suggestions for Further Analysis}

As pointed out, all methods for reconstructing large phylogenetic trees, i.e., trees with more than 10 to 15 taxa, try to contend with the enormous number of possible trees (Felsenstein, 1978) by heuristic search methods (Swof- ford et al., 1996). Doing so, none of these methods are guaranteed to find the globally optimal tree, i.e., the overall best tree. Each method has its advantages and drawbacks, which influence the result in a way that is not fully understood. Hence, "the one and only" method to reconstruct trees is not available. The authors suggest applying different methods to reconstruct trees, e.g., maximum-likelihood, maximum-parsimony, and distance-based methods. If all these methods provide the same tree topologies, then one may place some confidence in the results. If all these methods produce different tree topologies, one should interpret the data with great care and perform further analyses to find out what is going on (Sanderson and Shaffer, 2002).

In this context, TREE-PUZZLE can be used as a generator for data-driven plausible trees. For example, one can analyze the intermediate trees, which may be different from the consensus tree, to study the distribution of different trees, which provides an indication of noise in the data. Alternatively, one may use the set of intermediate trees to apply the tests outlined in the section about comparison of trees.

In conclusion, there is no one ideal method for phylogenetic analysis. Each dataset deserves its own careful analyses guided by the results of the rich collection of tree building methods (Swofford et al., 1996). And finally, remember that sometimes, a tree is simply not the best way to visualize the data.

\section{Literature Cited}

Adachi, J. and Hasegawa, M. 1996. Model of amino acid substitution in proteins encoded by mitochondrial DNA. J. Mol. Evol. 42:459-468.

Dayhoff, M.O., Schwartz, R.M., and Orcutt, B.C. 1978. A model of evolutionary change in proteins. In Atlas of Protein Sequence Structure, vol. 5 (M.O. Dayhoff, ed.) pp. 345-352. National Biomedical Research Foundation, Washington DC.

Edwards, A.W.F. and Cavalli-Sforza, L.L. 1964. Reconstruction of evolutionary trees. In Phenetic and Phylogenetic Classification (V.H. Heywood and J. McNeill, eds.) pp. 67-76. Systematics Association, London.

Felsenstein, J. 1978. The number of evolutionary trees. Syst. Zool. 27:27-33.

Felsenstein, J. 1981. Evolutionary trees from DNA sequences: A maximum likelihood approach. $J$. Mol. Evol. 17:368-376.

Felsenstein, J. 1984. Distance methods for inferring phylogenies: A justification. Evolution 38:16-24.

Goldman, N. 1993a. Statistical tests of models of DNA substitution. J. Mol. Evol. 36:182-198.
Inferring

Evolutionary

Relationships

6.6.21

Supplement 1 
Goldman, N. 1993b. Simple diagnostic statistical tests of models for DNA substitution. J. Mol. Evol. 37:650-661.

Goldman, N., Anderson, J.P., and Rodrigo, A.G. 2000. Likelihood-based tests of topologies in phylogenetics. Syst. Biol. 49:652-670.

Gu, X., Fu, Y.-X., and Li, W.-H. 1995. Maximum likelihood estimation of the heterogeneity of substitution rate among nucleotide sites. Mol. Biol. Evol. 12:546-557.

Hasegawa, M., Kishino, H., and Yano, T. 1985. Dating the human-ape split by a molecular clock of mitochondrial DNA. J. Mol. Evol. 22:160174.

Henikoff, S. and Henikoff, J.G. 1992. Amino acid substitution matrices from protein blocks. Proc. Natl. Acad. Sci. U.S.A. 89:10915-10919.

Hillis, D.M. 1996. Inferring complex phylogenies. Nature 383:130-131.

Iwabe, N., Kuma, K.-I., Hasegawa, M., Osawa, S., and Miyata, T. 1989. Evolutionary relationship of Archaebacteria, Eubacteria, and Eukaryotes inferred from phylogenetic trees of duplicated genes. Proc. Natl. Acad. Sci. U.S.A. 86:93559359.

Jones, D.T., Taylor, W.R., and Thornton, J.M. 1992. The rapid generation of mutation data matrices from protein sequences. Comput. Appl. Biosci. $8: 275-282$

Jukes, T.H. and Cantor, C.R. 1969. Evolution of protein molecules. In Mammalian Protein Metabolism (H.N. Munro, ed.). Academic Press, New York.

Kimura, M. 1980. A simple method for estimating evolutionary rates of base substitutions through comparative studies of nucleotide sequences. $J$. Mol. Evol. 16:111-120.

Kishino, H. and Hasegawa, M. 1989. Evolution of the maximum likelihood estimate of the evolutionary tree topologies from DNA sequence data, and the branching order in Hominoidea. J. Mol. Evol. 29:170-179.

McMorris, F.R. and Neumann, D.A. 1983. Consensus functions defined on trees. Math. Soc. Sci. 4:131-136

Müller, T. and Vingron, M. 2000. Modeling amino acid replacement. J. Comput. Biol. 7:761-776.

Page, R.D. and Holmes, E.C. 1998. Molecular Evolution: A Phylogenetic Approach. Blackwell Science, Oxford.

Posada, D. and Crandall, K.A. 1998. MODELTEST: Testing the model of DNA substitution. Bioinformatics 14:817-818.

Sanderson, M.J. and Shaffer, H.B. 2002. Troubleshooting molecular phylogenetic analyses. Annu. Rev. Ecol. Syst. 33:49-72.

Schmidt, H.A., Strimmer, K., Vingron, M., and von Haeseler, A. 2002. TREE-PUZZLE: Maximum likelihood phylogenetic analysis using quartets and parallel computing. Bioinformatics 18:502504.

Schöniger, M. and von Haeseler, A. 1994. A stochastic model for the evolution of autocorrelated DNA sequences. Mol. Phyl. Evol. 3:240-247.

Shimodaira, H. and Hasegawa, M. 1999. Multiple comparisons of log-likelihoods with applications to phylogenetic inference. Mol. Biol. Evol. 16:1114-1116.

Strimmer, K. and von Haeseler, A. 1996. Quartet puzzling: A quartet maximum likelihood method for reconstructing tree topologies. Mol. Biol. Evol. 13:964-969.

Strimmer, K. and von Haeseler, A. 1997. Likelihood mapping: A simple method to visualize phylogenetic content of a sequence alignment. Proc. Natl. Acad. Sci. U.S.A. 94:6815-6819.

Strimmer, K. and Rambaut, A. 2002. Inferring confidence sets of possibly misspecified gene trees. Proc. R. Soc. Lond. B 269:137-142.

Strimmer, K., Goldman, N., and von Haeseler, A. 1997. Bayesian probabilities and quartet puzzling. Mol. Biol. Evol. 14:210-213.

Swofford, D.L., Olsen, G.J., Waddell, P.J., and Hillis, D.M. 1996. Phylogeny reconstruction. In Molecular Systematics, 2nd ed. (D.M. Hillis, C. Moritz, and B.K. Mable, eds.) pp. 407-514. Sinauer Associates, Sunderland, Mass.

Tamura, K. and Nei, M. 1993. Estimation of the number of nucleotide substitutions in the control region of mitochondrial DNA in humans and chimpanzees. Mol. Biol. Evol. 10:512-526.

Whelan, S. and Goldman, N. 2001. A general empirical model of protein evolution derived from multiple protein families using a maximum likelihood approach. Mol. Biol. Evol. 18:691-699.

\section{Key References}

Goldman et al., 2000. See above.

A comprehensive review discussing tests for tree topologies and their applicability.

Page and Holmes, 1998. See above.

A well written textbook about phylogenetics and its applications.

Sanderson and Shaffer, 2002. See above.

A good review on problems in phylogeny reconstruction.

Strimmer and von Haeseler, 1996. See above.

An original publication of the Quartet Puzzling method.

Strimmer and von Haeseler, 1997. See above.

A more detailed description of Likelihood Mapping.

Swofford et al., 1996. See above.

An excellent introduction into the the rich collection of phylogenetic methods.
Maximum-

Likelihood Analysis Using TREE-PUZZLE

6.6 .22

Supplement 1 
Internet Resources

http://www.tree-puzzle.de

TREE-PUZZLE Web site.

http://rdp.cme.msu.edu/download/programs/ TreeTool/

TreeTool Web site (tree drawing program).

http://taxonomy.zoology.gla.ac.uk/ rpage/ treeview.html

TreeView Web site (tree drawing program, see UNIT 6.2).

http://evolution.genetics.washington.edu/phylip/ software.html
Joe Felsenstein's list of tree reconstruction and drawing programs.

http://www.ghostscript.com/

GhostScript Web page (PostScript viewer).

Contributed by Heiko A. Schmidt

Max-Planck-Institut für Molekulare Genetik

Berlin, Germany

Arndt von Haeseler

HHU Düsseldorf

Düsseldorf, Germany and

FZ Jülich

Jülich, Germany
Inferring

Evolutionary

Relationships

6.6.23

Supplement 1 